\title{
A large-aperture strip-grid beam splitter for partially combined two millimeter-wave diagnostics on Korea Superconducting Tokamak Advanced Research
}

Cite as: Rev. Sci. Instrum. 90, 014703 (2019); https://doi.org/10.1063/1.5066611

Submitted: 16 October 2018 . Accepted: 13 December 2018 . Published Online: 04 January 2019

D. J. Lee (D), W. Lee (D), H. K. Park, and T. G. Kim

\section{ARTICLES YOU MAY BE INTERESTED IN}

A cryogenic cylindrical ion trap velocity map imaging spectrometer

Review of Scientific Instruments 90, 013101 (2019); https://doi.org/10.1063/1.5079264

Contributed Review: A review of compact interferometers

Review of Scientific Instruments 89, 121501 (2018); https://doi.org/10.1063/1.5052042

A single-chip integrated transceiver for high field NMR magnetometry

Review of Scientific Instruments 90, 015001 (2019); https://doi.org/10.1063/1.5066436

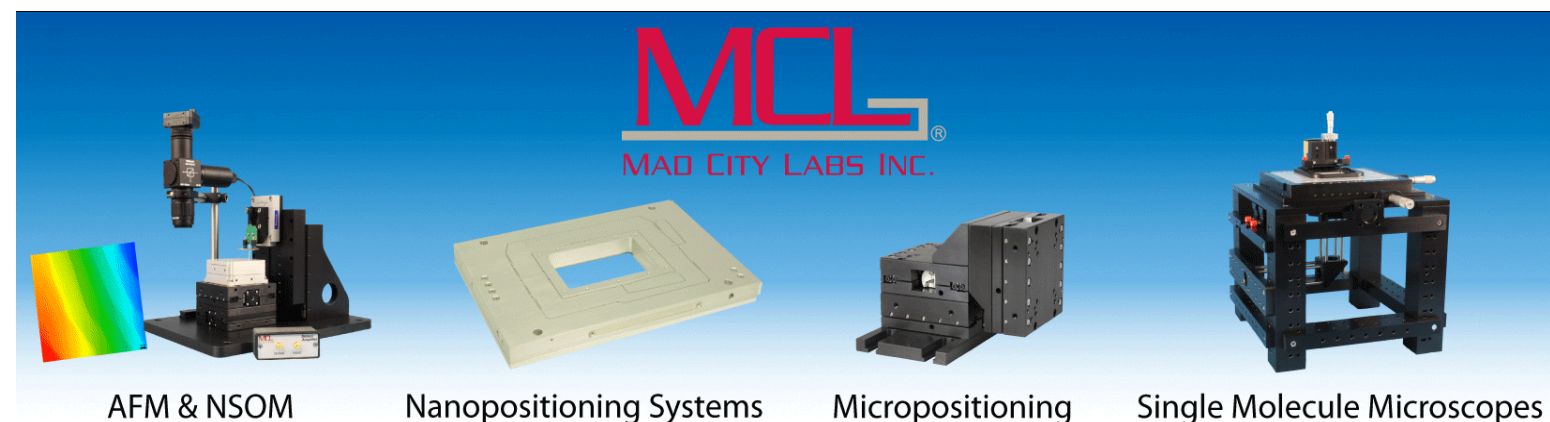




\title{
A large-aperture strip-grid beam splitter for partially combined two millimeter-wave diagnostics on Korea Superconducting Tokamak Advanced Research
}

\author{
Cite as: Rev. Sci. Instrum. 90, 014703 (2019); doi: 10.1063/1.506661 1 \\ Submitted: 16 October 2018 - Accepted: 13 December 2018 • \\ Published Online: 4 January 2019
}

D. J. Lee, ' (D) W. Lee, ${ }^{2, a)}$ (D) H. K. Park, ', and T. G. Kim ${ }^{3}$

\begin{abstract}
AFFILIATIONS
${ }^{1}$ Department of Physics, Ulsan National Institute of Science and Technology, Ulsan 44919, South Korea

${ }^{2}$ National Fusion Research Institute, Daejeon 34133, South Korea

${ }^{3}$ School of Electronics Engineering, Kyungpook National University, Daegu 41566, South Korea
\end{abstract}

a) Electronic mail: wclee@nfri.re.kr

\begin{abstract}
A large-aperture beam splitter has been developed for simultaneous operation of two millimeter-wave diagnostics employing different probe beams in the frequency and polarization, microwave imaging reflectometer ( 85 GHz X-mode), and collective scattering system (300 GHz O-mode), on the Korea Superconducting Tokamak Advanced Research device. The beam splitter was designed based on a polarizer concept (i.e., grid of metal strips on a thin dielectric sheet), and this can be an optimal solution for these two diagnostics. Fabrication of the strips with uniform sub-millimeter width and spacing on a large dielectric sheet was achieved with an etching technique, and the laboratory test results on the reflection and transmission ratio are in good agreement with design values.
\end{abstract}

Published under license by AIP Publishing. https://doi.org/10.1063/1.506661 1

\section{INTRODUCTION}

Several types of quasi-optical components have been employed in millimeter-wave imaging diagnostics on tokamaks: the large-aperture beam splitter, dichroic plate as a high-pass filter, ${ }^{1}$ notch filter for system protection, ${ }^{2,3}$ and half-wave plate for $90^{\circ}$ rotation of beam polarization. ${ }^{4}$ Among them, the beam splitter is widely used to split two beams for a single diagnostic or partially combined two diagnostics. Such beam splitters are used in the optical systems of two 2D millimeter-wave imaging diagnostics on the Korea Superconducting Tokamak Advanced Research (KSTAR) device: electron cyclotron emission imaging (ECEI) system $^{5}$ and microwave imaging reflectometer (MIR). ${ }^{6,7}$ For instance, large beam splitters are used to split ECE radiation for the dual-array ECEI system, and to split the probe beam and reflected beam for the MIR system. Another large beam splitter had been used to split ECE radiation and MIR beams for partially combined ECEI and MIR system until 2017. Small-size beam splitters (or power dividers) are used for the balanced split of beam power (of ECE radiation or MIR reflected beam or local oscillator beam) in the antenna/detector array box for odd-channel and evenchannel arrays. ${ }^{8}$ The large beam splitters were based on flat panel hard glass, $1.1 \mathrm{~mm}$ thick Borofloat 33, providing excellent flatness, which is the most critical optical element for $2 \mathrm{D}$ imaging diagnostics. On the other hand, the small beam splitters were made of a dielectric sheet, $0.5 \mathrm{~mm}$ thick RO4003C, showing an almost equal transmission and reflection ratio in a broad frequency range. In fact, the large beam splitters are power dividers like the small beam splitters so that only a half of the power is used, while the rest of the power is wasted. Especially, the MIR system had used two such splitters arranged in a row and consequently only $\sim 25 \%$ powers of the probe beam until 2017 and reflected beam were used for the MIR system. 
In the KSTAR tokamak, two millimeter-wave diagnostics, the MIR system and collective scattering system (CSS $)^{9}$ have been implemented on a single port prior to the 2018 campaign. Since the two diagnostics have to share the front optics, a large-aperture beam splitter is required to combine two probe beams (to the plasma) and split two return beams (from the plasma). Since the two diagnostic systems utilize entirely different probe beams in the frequency (78-96 $\mathrm{GHz}$ versus $300 \mathrm{GHz}$ ) and polarization (X-mode versus $\mathrm{O}$-mode), a wiregrid or strip-grid polarizer ${ }^{10}$ is potentially a good solution for effective division of the two beams with minimum power loss. The strip-grid polarizer on a thin dielectric substrate, $0.8 \mathrm{~mm}$ thick RO4003C, was chosen for the beam splitter instead of a wire-grid polarizer because it was difficult to find a manufacturer capable of making large-size wire-grid polarizers. Note that the substrate RO4003C is also used for notch filters to protect the detectors of the ECEI and MIR system from stray radiation of the high-power electron cyclotron resonant heating beam. ${ }^{11}$

The outline of this paper is as follows: In Sec. II, the principle of the strip-grid beam splitter on a thin dielectric substrate (and wire-grid splitter) is reviewed and an optimal design of the beam splitter for the two systems is given. In Sec. III, laboratory test results of the fabricated strip-grid beam splitter, including the reflection ratio of the $300 \mathrm{GHz} \mathrm{O}$-mode beam power and transmission ratio of the $78-96 \mathrm{GHz} \mathrm{X}$-mode beam power, are compared with the design values. Similar test results for the hard-glass beam splitter made of $1.1 \mathrm{~mm}$ thick Borofloat 33 are also compared with the design values. In particular, the reflection test result for the $300 \mathrm{GHz} O$-mode beam justifies why the new strip-grid beam splitter has to be used instead of the old hard-glass beam splitter. A summary follows in Sec. IV.

\section{REVIEW OF WORKING PRINCIPLE FOR OPTIMAL DESIGN}

Figure 1 illustrates the MIR system and CSS combined in a single port on KSTAR. Considering several issues such as a space limitation and higher sensitivity of the MIR system on the optical alignment, the MIR beams $(78-96 \mathrm{GHz}$ $\mathrm{X}$-mode) and CSS beams (300 $\mathrm{GHz} \mathrm{O}$-mode) were designed to be transmitted through and reflected from the first beam splitter (strip-grid beam splitter), respectively. In this case, the best option is that most of the $300 \mathrm{GHz} \mathrm{O}$-mode beam power is reflected from the beam splitter, while the $78-96 \mathrm{GHz}$ $\mathrm{X}$-mode beam power is transmitted as much as possible.

\section{A. Reflectance of the strip-grid beam splitter for the $300 \mathrm{GHz}$ O-mode beam}

When the electric field of an incident beam is parallel to the direction of metallic strips or wires, the impedance of the strip-grid with a width $2 a$ is given as ${ }^{12}$

$$
\frac{\mathrm{Z}_{\mathrm{g}}}{\mathrm{Z}_{\mathrm{fs}}}=j\left(\frac{g}{\lambda}\right) \ln \csc \left(\frac{\pi a}{g}\right)
$$

and the impedance of the wire-grid with a diameter $2 a$ is given as

$$
\frac{\mathrm{Z}_{\mathrm{g}}}{\mathrm{Z}_{\mathrm{fs}}}=j\left(\frac{g}{\lambda}\right) \ln \left(\frac{g}{2 \pi a}\right),
$$

where $Z_{\mathrm{g}}$ is the total grid impedance, $Z_{\mathrm{fs}}$ is the characteristic transmission line impedance, $g$ is the center-to-center grid spacing, $\lambda$ is the wavelength of the incident beam, and csc is the cosecant. Note that the impedances in Eqs. (1) and (2) are complex numbers. When the strips or wires are lossless, the electric field reflection coefficient has the form

$$
r_{\|}=\frac{E_{\text {ref }}}{E_{\text {inc }}}=\frac{-1}{1+2 Z_{\mathrm{g}} / Z_{\mathrm{fs}}},
$$

where $E_{\text {ref }}$ and $E_{\text {inc }}$ are the electric field of the reflected and incident beams, respectively. The reflectance (the ratio of reflected power to incident power) is the squared magnitude of the expression given in Eq. (3). The reflectance as a function of $g / \lambda$ with $2 a=0.15 \mathrm{~mm}$ is illustrated in Fig. 2 . We chose $2 a=0.15 \mathrm{~mm}$ (minimum available width) and $g=0.3 \mathrm{~mm}$ for our beam splitter, and the expected reflectance is $96 \%$ for the $300 \mathrm{GHz}$ incident beam.

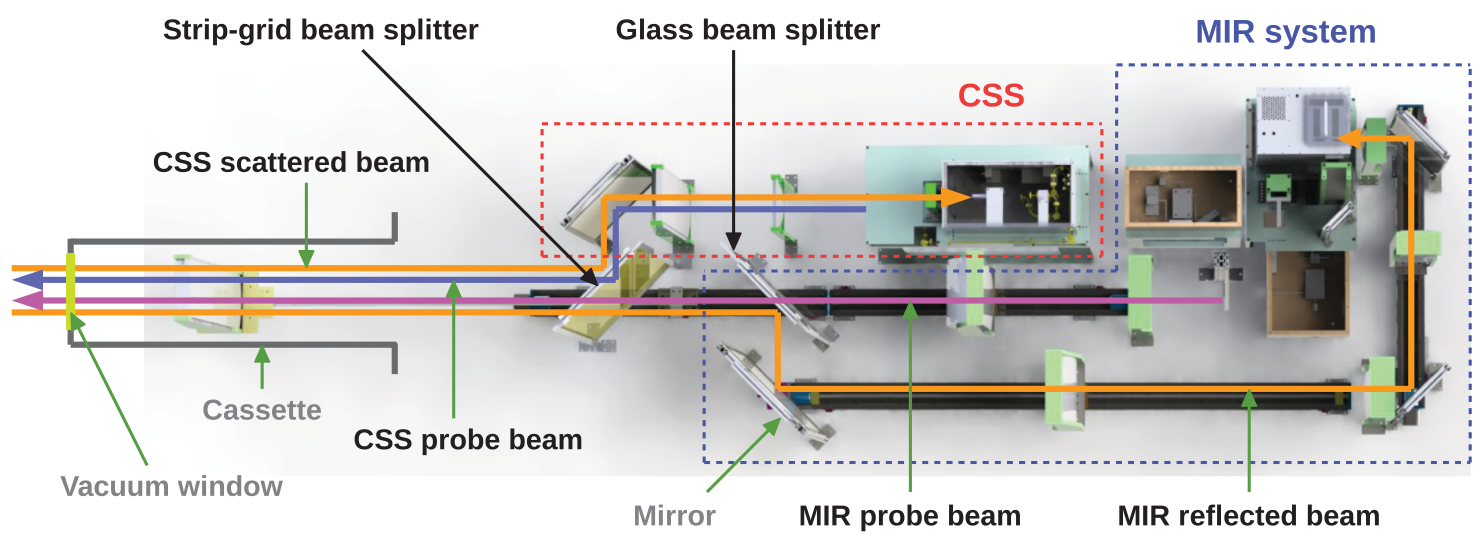

FIG. 1. A 3D drawing (top view) of the MIR system and CSS combined in a single port on KSTAR. 


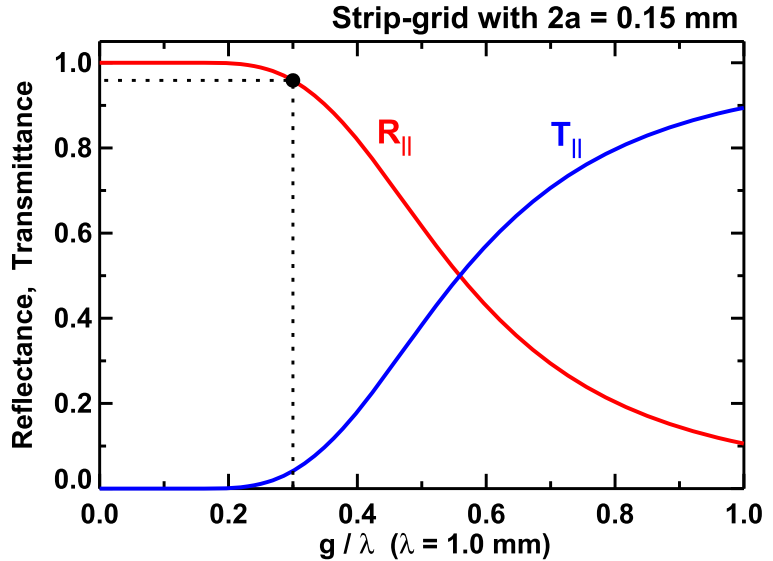

FIG. 2. The calculated reflectance (power reflection ratio) and transmittance (power transmission ratio) as a function of $g / \lambda$ of the strip-grid beam splitter with the strip width $2 a=0.15 \mathrm{~mm}$ for the $300 \mathrm{GHz}$ incident beam, in the case that the electric field direction of the incident beam is parallel to the direction of the strips. Here, $g$ is the center-to-center strip spacing and $\lambda$ is the wavelength of the incident beam. We chose $g=0.3 \mathrm{~mm}$ for our beam splitter and the expected reflectance is $96 \%$

\section{B. Transmittance of the dielectric substrate for the 78-96 GHz X-mode beam}

In the case of the perpendicular injection, the transmittance (the ratio of transmitted power to incident power) of the 78-96 GHz X-mode beam through the dielectric substrate is almost unity. However, the strip-grid beam splitter is positioned with $45^{\circ}$ in the horizontal direction, and then, the transmittance is not unity any more. The reflectance of a beam through a thin dielectric sheet is given as ${ }^{13}$

$$
\mathrm{R}=\frac{r_{12}^{2}+r_{23}^{2}+2 r_{12} r_{23} \cos \left(2 k_{0} N_{r} t \cos \theta_{2}\right)}{1+r_{12}^{2} r_{23}^{2}+2 r_{12} r_{23} \cos \left(2 k_{0} N_{r} t \cos \theta_{2}\right)},
$$

where $k_{0}$ is the wavenumber of the incident beam, $N_{r}$ is the real value of the complex refractive index $\left(N_{r}-j N_{i}\right)$ of the sheet, and $t$ is the thickness. The refractive index is related to the dielectric constant $\epsilon_{r}$ as $\mathrm{N}_{r}=\sqrt{\epsilon_{r}} \cdot r_{12}$ is the reflection coefficient of the beam field at surface 1 (front surface) defined as

$$
\begin{aligned}
& r_{12}^{\mathrm{s}}=-\frac{\sin \left(\theta_{1}-\theta_{2}\right)}{\sin \left(\theta_{1}+\theta_{2}\right)} \quad(\mathrm{S}-\text { polarization }) \\
& r_{12}^{p}=\frac{\tan \left(\theta_{1}-\theta_{2}\right)}{\tan \left(\theta_{1}+\theta_{2}\right)} \quad(\mathrm{P}-\text { polarization })
\end{aligned}
$$

and $r_{23}$ is the reflection coefficient of the beam field at surface 2 (rear surface) defined as

$$
\begin{gathered}
r_{23}^{\mathrm{s}}=-\frac{\sin \left(\theta_{2}-\theta_{3}\right)}{\sin \left(\theta_{2}+\theta_{3}\right)} \quad(\mathrm{S}-\text { polarization }), \\
r_{23}^{p}=\frac{\tan \left(\theta_{2}-\theta_{3}\right)}{\tan \left(\theta_{2}+\theta_{3}\right)} \quad(\mathrm{P}-\text { polarization }),
\end{gathered}
$$

where $\theta_{1}$ is the incident angle to the sheet surface $1, \theta_{2}$ is the refracted angle from the surface 1 (or the incident angle to the surface 2), and $\theta_{3}$ is the refracted angle from the surface 2. Note that the reflection coefficients are different for two linear polarizations: P-polarization (in the plane of incidence) and S-polarization (orthogonal to the plane of incidence). The dielectric loss of the beam power in the sheet, referred to as absorptance, is related to the imaginary value $\epsilon_{i}$ of the complex dielectric constant and has the form

$$
A=1-\exp \left(-\delta k_{0} N_{r} t\right)
$$

where the ratio of the imaginary to real dielectric constant, $\tan \delta=\epsilon_{i} / \epsilon_{r} \approx \delta(\ll 1)$, is the loss tangent. Then, the transmittance is given as

$$
\mathrm{T}=1-\mathrm{R}-\mathrm{A} .
$$

The dielectric constant and loss tangent of RO4003C are $3.38 \pm 0.05$ and $2.7 \times 10^{-3}$ at $10 \mathrm{GHz}$, respectively, and these values were used for the calculation over the frequency range from 75 to $110 \mathrm{GHz}$. This is because the values in the range from 75 to $110 \mathrm{GHz}$ have not been reported yet. If there is any significant discrepancy between the measured and calculated results in the frequency range, the uncertainty in the two parameters could be the primary source. Figure 3 illustrates the calculated transmittance as a function of the beam frequency of the substrate RO4003C with several available thicknesses from 0.2 to $0.8 \mathrm{~mm}$. The incident beam is S-polarized, and the incident angle is $45^{\circ}$. Between two thicknesses of $0.2 \mathrm{~mm}$ and $0.8 \mathrm{~mm}$ showing higher transmittance, we chose the $0.8 \mathrm{~mm}$ thickness because the thicker sheet provides better surface flatness. In addition, the uneven transmittance for the $0.8 \mathrm{~mm}$ thickness (higher transmittance at higher frequency) may partly balance out the uneven power loss in the $20 \mathrm{~m}$ long broadband cables (higher loss at higher frequency) between the MIR detector array and electronic system, and consequently enables final signal levels of four radial channels to be relatively even. The power loss in the broadband cable, including two short semi-rigid cables at the ends, is $\sim 3.5 \mathrm{~dB}$ at the intermediate frequency (IF) of $1.0 \mathrm{GHz}$ and $\sim 10 \mathrm{~dB}$ at

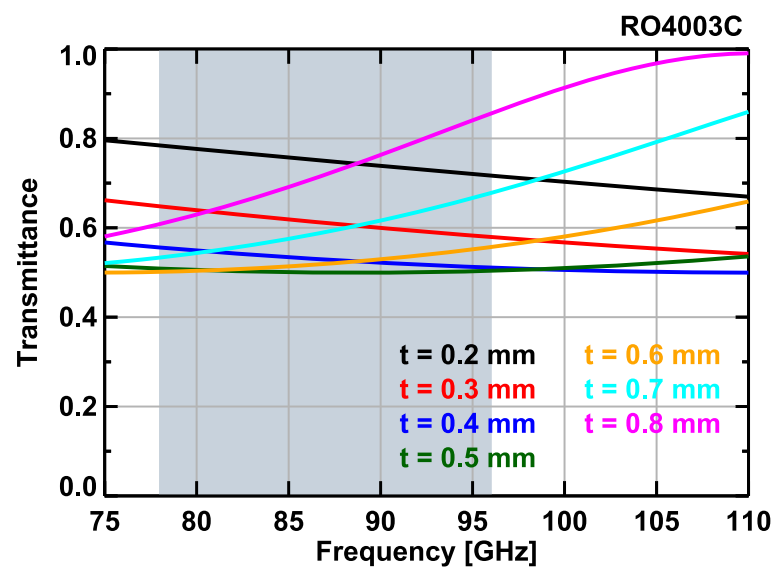

FIG. 3. The calculated transmittance as a function of frequency of the dielectric substrate RO4003C with several available thicknesses from 0.2 to $0.8 \mathrm{~mm}$. The incident beam is S-polarized, and the incident angle is $45^{\circ}$. The shaded region indicates the frequency range of the MIR probe beam $(78-96 \mathrm{GHz})$. 
IF $=6.4 \mathrm{GHz} .^{7}$ Here, higher IF corresponds to higher probing frequency.

\section{LABORATORY TEST}

Figure 4(a) is a picture of the fabricated strip-grid beam splitter (by EM-Wise Communications) with the strip width of $2 a=0.15 \mathrm{~mm}$ and center-to-center spacing of $g=0.3 \mathrm{~mm}$ on the $0.8 \mathrm{~mm}$ thick RO4003C substrate. The thickness of the copper strips is $17 \mu \mathrm{m}$. The uniform width and spacing of the strips on the large substrate were achieved with an etching technique. Figure 5 shows the test results of the beam splitter for the $300 \mathrm{GHz} \mathrm{O}$-mode beam together with the schematic diagrams of test setups. 2D beam intensity profiles were measured at a fixed distance of $Z=500 \mathrm{~mm}$ from the beam source for three cases: the incident beam without the beam splitter, the reflected beam from the beam splitter, and the transmitted beam through the beam splitter. Comparing the total beam powers, integrated over the 2D plane, for the three cases, the reflectance is $98.3 \%$ and the transmittance is $2.5 \%$. The measured reflectance is slightly higher by $\sim 2 \%$ than the design value (95.9\%). This is mostly due to the experimental errors such as beam power variation. The $300 \mathrm{GHz}$ beam power can slowly vary in time by $\sim 4 \%$ probably due to unstable bias voltages or temperature change. Note that the $300 \mathrm{GHz}$ beam source consists of a $12.5 \mathrm{GHz}$ synthesizer and $\times 24$ multiplier chain (made up of three doublers and a tripler), and the output powers are sensitive to the externally applied bias voltages. Figure 6(a) shows the measured and calculated reflectance, transmittance, and absorptance as a function of frequency from 75 to $110 \mathrm{GHz}$ of the strip-grid beam splitter. The measured results are best matched with the calculated results with the thickness of $0.84 \mathrm{~mm}$. The transmittance in the frequency range of the MIR probe beam $(78-96 \mathrm{GHz})$ is $65 \%-92 \%$.

As already mentioned in Sec. I, the beam splitter used for the division of the MIR probe beam and reflected beam was made of a hard glass, $1.1 \mathrm{~mm}$ thick Borofloat 33 . Figure 4(b) shows a newly fabricated hard-glass beam splitter for the modified MIR optical system to be combined with the CSS optical system. The measured and calculated reflectance, transmittance, and absorptance as a function of frequency of the beam splitter are shown in Fig. 6(b). The dielectric constant is 4.6 , and the loss tangent is $3.7 \times 10^{-3}$ at $1 \mathrm{MHz}$, which were used in the calculation over the frequency range from 75 to $110 \mathrm{GHz}$. As mentioned before, the small discrepancy between the measured and calculated results could be attributed to uncertainty in the two parameters over the frequency range, which is significantly higher than $1 \mathrm{MHz}$.

One important reason why the wire-grid or strip-grid beam splitter was considered for division of the MIR beams and CSS beams is that the reflectance of the $300 \mathrm{GHz} \mathrm{O}-$ mode beam from the hard-glass beam splitter is too low. Figure 7 shows the measured and calculated transmittance, reflectance, and absorptance of the hard-glass beam splitter for the $300 \mathrm{GHz} \mathrm{O}$-mode (P-polarized) beam. The measurement results are significantly different from the calculated results with the dielectric constant and loss tangent at $1 \mathrm{MHz}$, meaning that the two quantities at $1 \mathrm{MHz}$ cannot be used for the calculation at such a high frequency. The measurement results are matched with the calculated ones with a reduced dielectric constant 4.35 and largely increased loss tangent $2.7 \times 10^{-2}$. The measured reflectance of the hard-glass beam splitter for the $300 \mathrm{GHz} \mathrm{O}$-mode beam is only $13 \%$, which
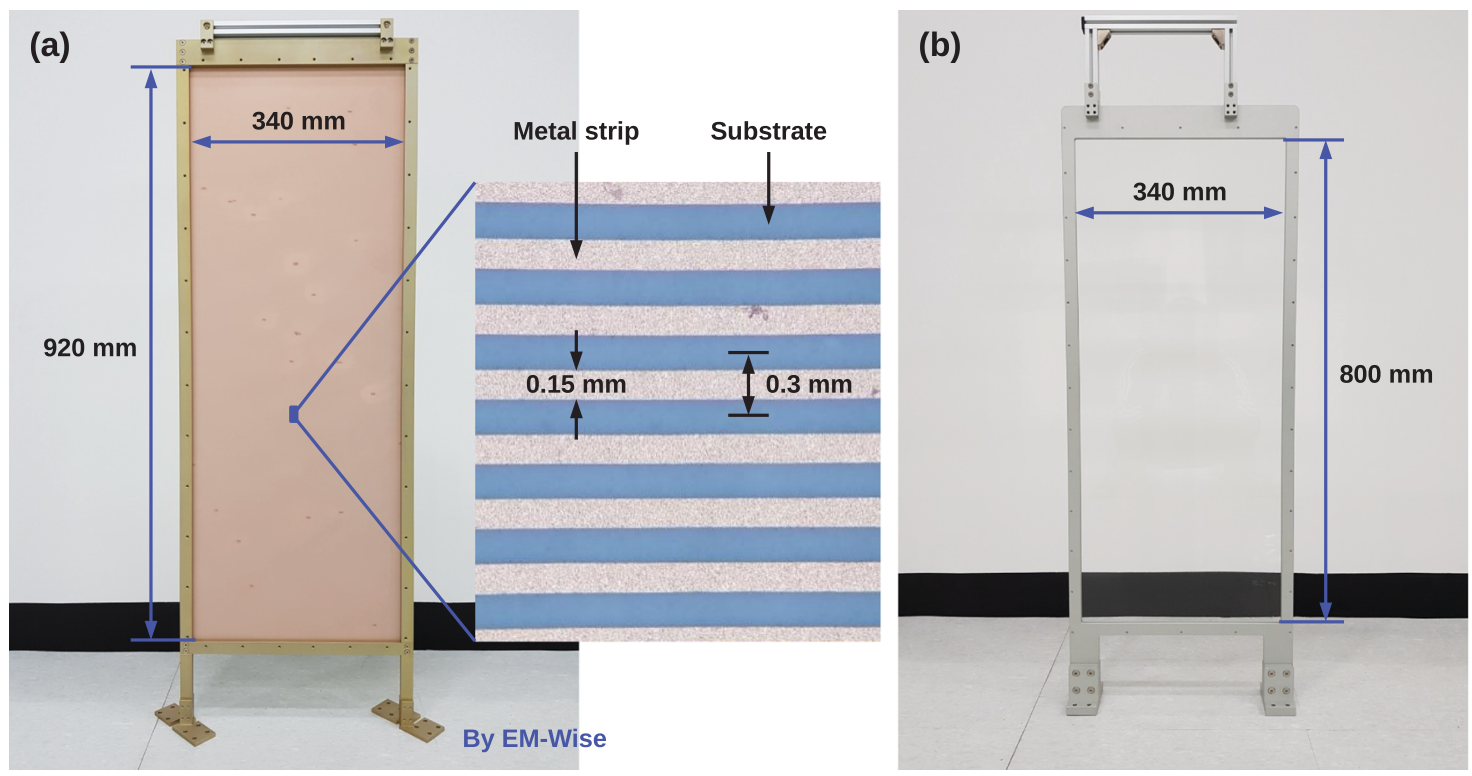

FIG. 4. (a) The fabricated strip-grid beam splitter with the strip width of $0.15 \mathrm{~mm}$ and center-to-center spacing of $0.3 \mathrm{~mm}$ on the $0.8 \mathrm{~mm}$ thick RO4003C substrate. The thickness of the copper strips is $17 \mu \mathrm{m}$. (b) A hard-glass beam splitter made of $1.1 \mathrm{~mm}$ thick Borofloat 33 . 
(a) Incident beam
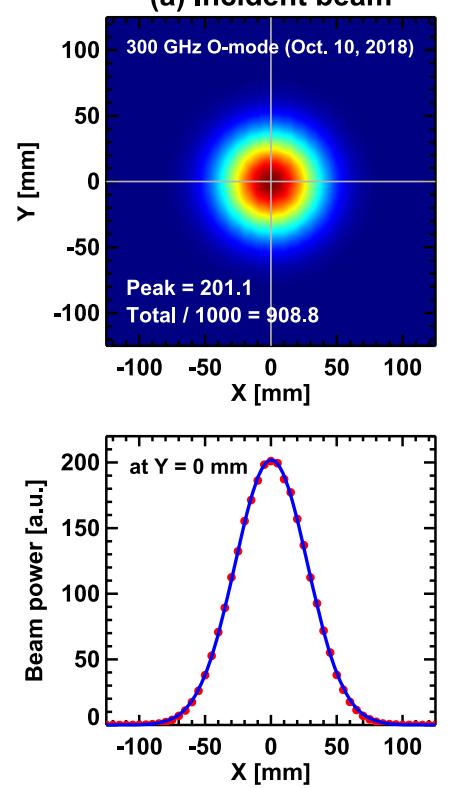

(d)

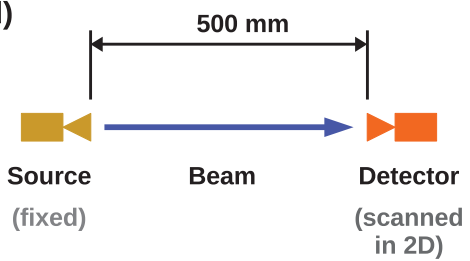

(Top view) (b) Reflected beam
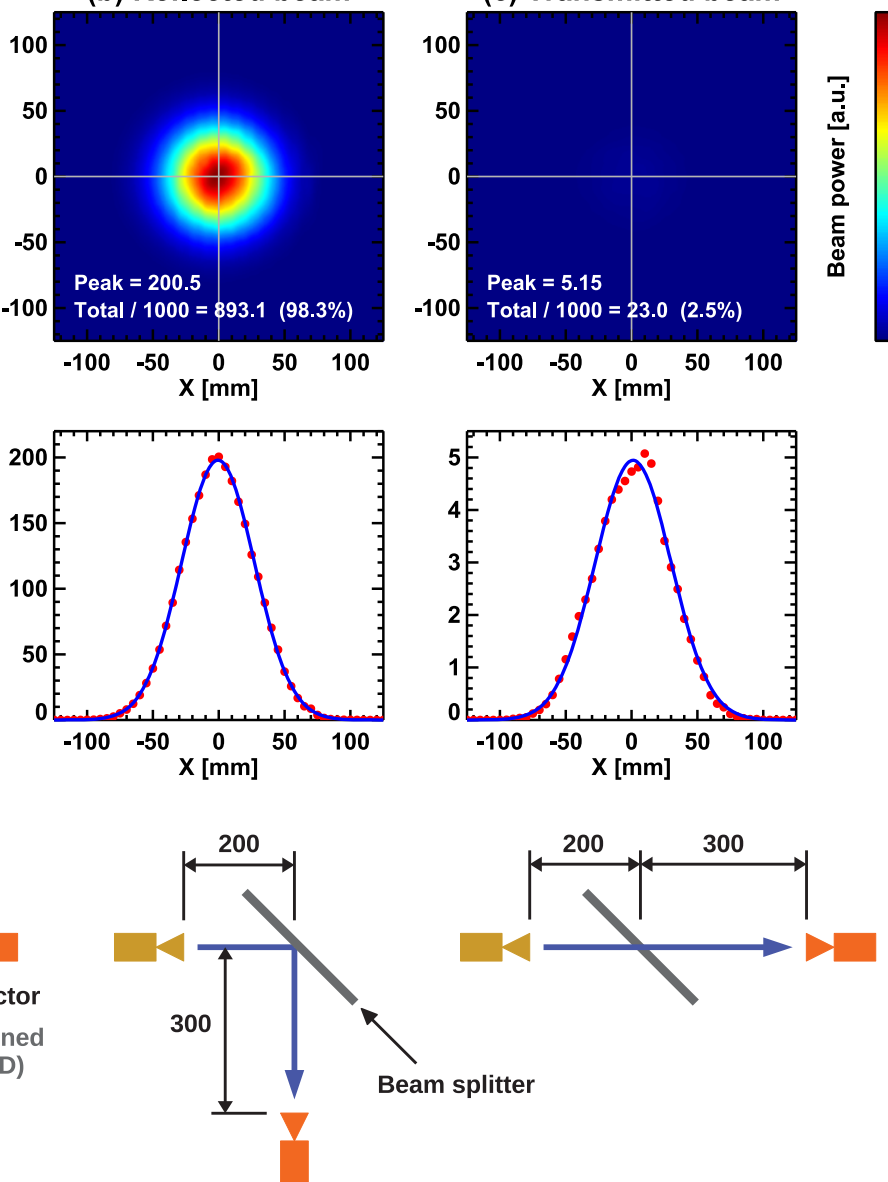

(c) Transmitted beam
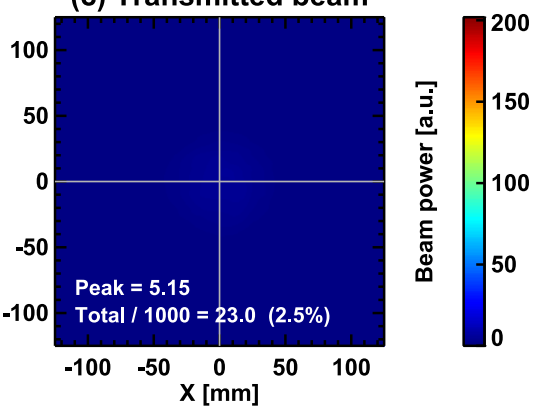

FIG. 5. The measured 2D and 1D intensity profiles of three $300 \mathrm{GHz}$ O-mode beams: (a) the incident beam without the strip-grid beam splitter, (b) the reflected beam from the beam splitter, and (c) the transmitted beam through the beam splitter. The axial distance from the source to the detection plane was $500 \mathrm{~mm}$, and the beam splitter was positioned at $200 \mathrm{~mm}$ from the source. (d) Schematic diagrams of the three measurement setups.
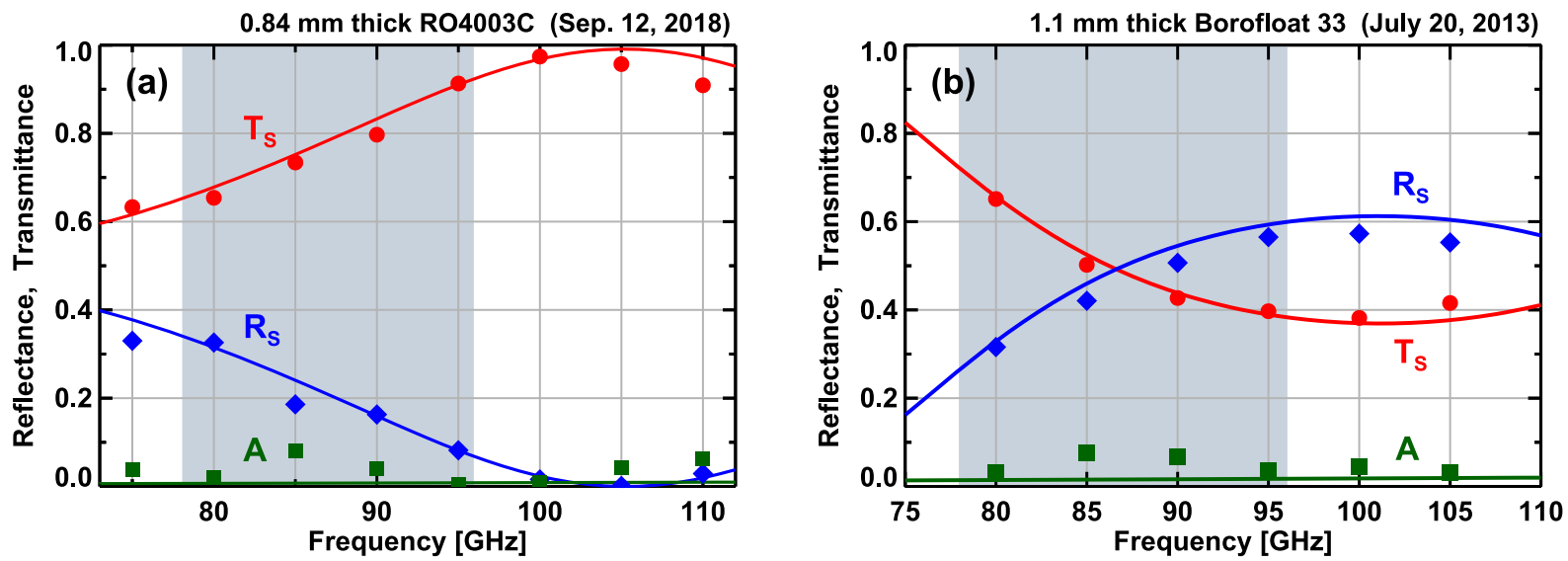

FIG. 6. (a) The measured (symbols) and calculated (curves) transmittance, reflectance, and absorptance as a function of frequency from 75 to $110 \mathrm{GHz}$ of the strip-grid beam splitter for the S-polarized beam. The measured results are best matched with the calculated curves with the thickness of $0.84 \mathrm{~mm}$. (b) The measured (symbols) and calculated (curves) transmittance, reflectance, and absorptance as a function of frequency of the hard-glass beam splitter. The shaded region indicates the frequency range of the MIR probe beam $(78-96 \mathrm{GHz})$. Note that the measured absorptance was obtained using the relationship $A=1-T_{\mathrm{S}}-R_{\mathrm{S}}$ and measured $T_{\mathrm{S}}$ and $R_{\mathrm{S}}$. 
$1.1 \mathrm{~mm}$ thick Borofloat 33 (March 21, 2016)

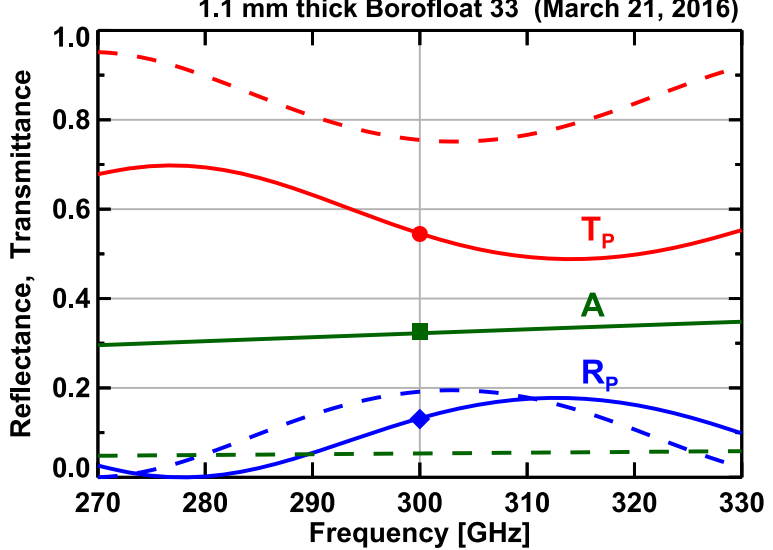

FIG. 7. Transmittance, reflectance, and absorptance of the hard-glass beam splitter for the P-polarized beam: measured at $300 \mathrm{GHz}$ (symbols) and calculated in $270-330 \mathrm{GHz}$ (curves). The solid curves were obtained with the dielectric constant 4.35 and loss tangent $2.7 \times 10^{-2}$, and the dashed curves with 4.6 and $3.7 \times 10^{-3}$, which are the reported values at $1 \mathrm{MHz}$.

is significantly lower than $\sim 96 \%$ of the new strip-grid beam splitter.

\section{SUMMARY}

A large-aperture beam splitter has been developed for simultaneous operation of the partially combined MIR system and CSS to optimize the return beam as well as the scattered beam. Since the two diagnostics employ entirely different probe beams in the frequency and polarization, a wire-grid or strip-grid polarizer is an optimal solution. The fabricated strip-grid beam splitter with the strip width of $0.15 \mathrm{~mm}$ and center-to-center spacing of $0.3 \mathrm{~mm}$ on a $0.8 \mathrm{~mm}$ thick RO4003C substrate shows $\sim 96 \%$ reflectance for the $300 \mathrm{GHz} \mathrm{O}$-mode beam (for the CSS) and 65\%-92\% transmittance for the 78-96 GHz X-mode beam (for the MIR system), which agree with the design values. The choice of the $0.8 \mathrm{~mm}$ thickness provides better surface flatness and uneven transmittance in the range of 78-96 GHz (higher transmittance at higher frequency), and the latter property may contribute to relatively even final signal levels of four radial channels of the MIR system. A hard-glass beam splitter made of $1.1 \mathrm{~mm}$ thick Borofloat 33, used for split of the MIR probe beam and reflected beam from 2013, has been newly fabricated for the modified MIR optical system.

\section{ACKNOWLEDGMENTS}

This research was supported by the Ministry of Science, ICT and Future Planning of Korea under the KSTAR project and the National Research Foundation of Korea under Contract Nos. NRF-2014M1A7A1A03029865 and NRF2015M1A7A1A02002627.

\section{REFERENCES}

${ }^{1}$ G. Bertschinger, C. P. Endres, F. Lewen, and J. W. Oosterbeek, Rev. Sci Instrum. 79, $10 \mathrm{E} 709$ (2008).

${ }^{2}$ Z. Shen, N. Ito, E. Sakata, C. W. Domier, Y. Liang, N. C. Luhmann, Jr., and A. Mase, "Frequency selective surface notch filter for use in a millimeter wave imaging system," in Proceedings of 2006 IEEE Antennas and Propagation Society International Symposium (IEEE, 2006), p. 4191.

${ }^{3}$ Z. Shen, N. Ito, Y. Liang, L. Lin, C. W. Domier, M. Johnsaon, N. C. Luhmann, Jr., A. Mase, and E. Sakata, Plasma Fusion Res. 2, S1030 (2007).

${ }^{4}$ J. Lee, G. S. Yun, M. Kim, W. Lee, and H. K. Park, J. Instrum. 7, C01037 (2012).

${ }^{5}$ G. S. Yun, W. Lee, M. J. Choi, J. Lee, M. Kim, J. Leem, Y. Nam, G. H. Choe, H. K. Park, H. Park, D. S. Woo, K. W. Kim, C. W. Domier, N. C. Luhmann, Jr. N. Ito, A. Mase, and S. G. Lee, Rev. Sci. Instrum. 85, $11 \mathrm{D} 820$ (2014).

${ }^{6}$ W. Lee, J. Leem, J. A. Lee, Y. B. Nam, M. Kim, G. S. Yun, H. K. Park, Y. G. Kim, H. Park, K. W. Kim, C. W. Domier, N. C. Luhmann, Jr., K. D. Lee, Y. U. Nam, W. H. Ko, J. H. Jeong, Y. S. Bae, and KSTAR Team, Nucl. Fusion 54, 023012 (2014).

${ }^{7}$ W. Lee, J. Leem, D. J. Lee, M. J. Choi, H. K. Park, J. A. Lee, G. S. Yun, T. G. Kim, H. Park, K. W. Kim, and KSTAR Team, Plasma Phys. Controlled Fusion 60 115009 (2018).

${ }^{8}$ T. Munsat, C. W. Domier, X. Kong, T. Liang, N. C. Luhmann, Jr., B. J. Tobias, W. Lee, H. K. Park, G. Yun, I. G. J. Classen, and A. J. H. Donne, Appl. Opt. 49, E20 (2010)

${ }^{9}$ W. Lee, H. K. Park, D. J. Lee, Y. U. Nam, J. Leem, and T. K. Kim, Rev. Sci. Instrum. 87, 043501 (2016).

${ }^{10}$ T. Kondo, T. Nagashima, and M. Hangyo, Jpn. J. Appl. Phys., Part 2 42, L373 (2003).

${ }^{11}$ W. Mohyuddin, D. S. Woo, S. K. Kim, K. W. Kim, and H.-C. Choi, J. Sens. Sci. Technol. 25, 8 (2016).

${ }^{12}$ P. F. Goldsmith, Quasioptical System: Gaussian Beam Quasioptical Propagation and Apllications (Wiley-IEEE Press, 1998), p. 235.

${ }^{13}$ W. J. Smith, Modern Optical Engineering: The Design of Optical Systems, 2nd ed. (McGraw-Hill, 1990), p. 187. 